\title{
Rain, rain, rain
}

\section{Malcolm Walker}

Monsoons. Edited by Jay S. Fein and Pamela L. Stephens. Wiley: 1987. Pp. 632. £71.75, \$104.95.

DeRIVED from the Arabic word mawsim, meaning fixed season, the term monsoon refers strictly to the seasonal nature of the winds which blow over southern Asia, Indonesia, northern Australia, the Arabian Sea, the Bay of Bengal, the South China Sea and much of North Africa. Popularly, however, the word refers also to the summer rains of these regions.

In all monsoonal regions, abrupt changes in atmospheric behaviour take place at specific times of year. Characteristic of the onset and retreat phases of monsoons, these changes involve comprehensive and widespread reorganizations of atmospheric circulation patterns. Reversals of wind direction occur at all levels in the troposphere and lower stratosphere; and associated changes in patterns of vertical motion are such that the droughts of winter are replaced by wet weather in summer. Rainfall varies markedly from year to year. In some years it is widespread and abundant. In others it is scanty. It is always unreliable. Sometimes the rains fail at a critical time; and sometimes there are short bursts of torrential rain rather than the periods of steady rain preferred by farmers.

Monsoons are of enormous social, agricultural and economic importance, and they dominate the lives of more than a thousand million people. For these people there are vital questions. Will the monsoon be early or will it be late? Will there be too much rain or too little? Meteorologists are interested in these questions too, and they seek answers to related questions, partly for altruistic reasons and partly in response to a purely scientific challenge. What drives monsoons? Why do they vary so much from year to year? Can they be forecast? How do they interact with atmospheric circulations outside the tropics?

Natural philosophers have long been fascinated and occupied with monsoons, particularly that of southern Asia, the most impressive of all seasonal phenomena. Indeed, the classical thermal concept, which likens a monsoon to a giant sea breeze, and which is still widely accepted even though its shortcomings have been recognized for many years, was first proposed as long ago as 1686 , by Edmund Halley, in a paper published in The Philosophical Transactions of the Royal Society of London.

Until about 30 years ago reliable investigations of monsoons were impossible because of the paucity of upper-air data and the consequent need to base the study of tropical weather systems largely upon surface data. Since then, however, techniques have been developed which enable us to reach well into the stratosphere. Moreover, satellites have provided a new source of data, and computer power to process the data has increased greatly. There has also been a growth of interest in the tropical atmosphere, from which monsoon research has benefited; and a number of special research enterprises involving international collaboration have been mounted in monsoonal regions (the International Indian Ocean Expedition of 1959-1965, the IndoSoviet Monsoon Experiment of 1973 and the International Monsoon Experiment of 1977-1979, for example). Advances in monsoon meteorology have been rapid in recent years, so it was appropriate to pause and take stock of current knowledge.

This book is more than a scientific review, however, for it is concerned not only with atmospheric processes and the wind-driven circulations of the Indian Ocean but also with various other facets of monsoons. There are, for example, three chapters devoted to the literature and folklore of India; these accounts deal with poetry and prose, proverbs, symbolism, religion, rituals, festivals and medical regimens, and they demonstrate the enormous and continuing influence of the south-west monsoon on virtually every aspect of traditional culture in India. There is, too, a chapter on ancient and mediaeval records of the monsoon winds and currents of the Indian Ocean; and in another contribution the evolution of monsoon meteorology is traced from the seventeenth century to the twentieth. Other chapters concentrate upon the economic implications of weather and climate in monsoonal regions, the economic consequences of abnormal monsoons, and the strategies and programmes by means of which governments might deal with the aftermath of such monsoons.

Ten of the 19 chapters are devoted to meteorology, with the emphasis upon the vagaries and variability of monsoons on various time scales. One chapter is concerned with interannual and intraseasonal variability, for example, and another considers the evidence for and possible causes of secular changes in the timing, extent and strength of monsoons. The problem of prediction also receives a great deal of attention. The characteristics, applicability and limitations of various types of numerical models are discussed at length; and two chapters focus upon the mix of synoptic, empirical and numerical techniques used operationally by weather forecasters in India and South East Asia.

It is clear from the book that monsoons are meteorologically much more complex than was supposed only three decades

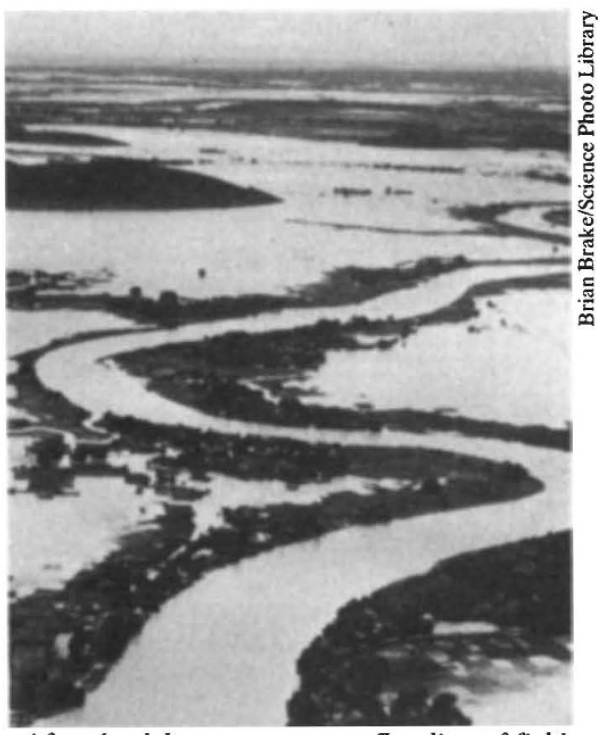

After the deluge - monsoon flooding of field systems on the plains of India.

ago, let alone in Halley's time. It is also clear that a complete understanding of them cannot be achieved without a thorough grasp not only of the underlying causes of atmospheric circulations and the relevant physics, but also of orographic influences, the effects of sea-surface temperature anomalies, and the teleconnections between monsoons and atmospheric events far from monsoonal regions. Indeed, monsoons are evidently not so much regional phenomena as elements in the global ocean-atmosphere system. Numerical modelling of tropical weather systems has plainly not yet advanced much beyond the experimental stage, and further progress in monsoon forecasting on all time scales will depend upon improved models and even greater computer power. More data will also be needed, as observations, unlike ideas and models, are still not plentiful.

The book is intended for meteorologists, climatologists, oceanographers, environmental scientists, folklorists, administrators and policymakers, and it takes the form of a collection of essays, each written by a distinguished expert. The 16 authors - and the editors too have acquitted themselves well. The style is lucid and fluent throughout; every chapter includes an extensive bibliography; numerous illustrations and tables of data are provided; and the standard of presentation is consistently high.

Although the approach is largely nonmathematical, a familiarity with the physics of fluids is required to follow some of the meteorology. Nevertheless, the general reader should not be deterred. As claimed in the blurb, this volume will be an "an ideal sourcebook for anyone interested in a phenomenon that so greatly affects so much of mankind".

Malcolm Walker is a Senior Lecturer in the Department of Maritime Studies, UWIST, Cardiff CF1 $3 E U, U K$. 